\title{
Complementation in vitro Between guaB Mutants of Escherichia coli $\mathrm{K} 12$
}

\author{
By H. J. GILBERT AND W. T. DRABBLE* \\ Department of Biochemistry, University of Southampton, Southampton SO9 $3 T U$
}

(Received 28 June 1979; revised 3 September 1979)

\begin{abstract}
Guanine auxotrophs of Escherichia coli were isolated following mutagenesis by $N$-methyl$N^{\prime}$-nitro- $N$-nitrosoguanidine or ethyl methanesulphonate. The mutants were classified according to growth properties and absence of IMP dehydrogenase or GMP synthetase activity. Mutations in guaB (IMP dehydrogenase-less) were analysed by reversion and suppression tests; all were of the base substitution missense type except for one possible frameshift and one polar nonsense mutation. GuaB mutants were examined for protein (CRM) that cross-reacts with monospecific antibodies to IMP dehydrogenase; approximately half were $\mathrm{CRM}^{+}$. Enzyme complementation in vitro was detected in mixed denatured and renatured cell-free extracts of any CRM ${ }^{+}$guaB mutant and PL1138 (guaB105, CRM ${ }^{+}$); $\mathrm{CRM}^{-}$mutants did not complement. GuaB105 maps distal to all other guaB mutations except guaB86 (CRM-). Two hybrid enzymes produced by complementation were less stable to heat than native IMP dehydrogenase, although kinetic constants were similar. These observations indicate interallelic complementation between guaB mutants and are consistent with the demonstration of identical subunits for IMP dehydrogenase (Gilbert et al., 1979). Only the subunits supplied by PL1138 are catalytically active in the hybrid enzymes suggesting that this mutant may produce a repairable polypeptide whereas the enzymes of complementing mutants may be defective at the active site.
\end{abstract}

\section{INTRODUCTION}

The biosynthesis of guanosine $5^{\prime}$-monophosphate (GMP) from inosine $5^{\prime}$-monophosphate (IMP) involves two enzymes. IMP dehydrogenase catalyses the conversion of IMP to xanthosine 5'-monophosphate (XMP) and GMP synthetase the conversion of XMP to GMP. IMP dehydrogenase and GMP synthetase are the products of the two structural genes, guaB and guaA respectively, of the gua operon (Lambden \& Drabble, 1973). As IMP dehydrogenase catalyses the first reaction unique to guanine nucleotide biosynthesis it plays an important role in controlling the production of GMP by feedback inhibition and by enzyme repression (Mager \& Magasanik, 1960; Nijkamp \& DeHaan, 1967). The enzyme from Escherichia coli has been studied in some detail and this has resulted in conflicting reports about its subunit structure. Krishnaiah $(1975)$ and Gilbert et al. $(1978,1979)$ have proposed identical subunits whereas Powell (1973) suggested non-identical subunits.

Complementation, the restoration of biological activity by the non-covalent interaction of different polypeptides, can be used to determine the nature of gene products, in particular the number of subunit types in oligomeric proteins. Complementation can be either intercistronic or interallelic (intracistronic). The former involves the interaction of distinct polypeptide chains each specified by its own cistron, as for example, with tryptophan synthetase of $E$. coli. The latter involves interactions between polypeptides from the same cistron and has been demonstrated for several enzymes including alkaline phosphatase and glutamate dehydrogenase (for a general review, see Zabin \& Villarejo, 1975).

This report describes the isolation and characterization of guaB (IMP dehydrogenase- 
less) mutants of $E$. coli. Complementation in vitro between the inactive enzymes of these strains is consistent with the presence of identical subunits in IMP dehydrogenase.

\section{METHODS}

Bacterial strains. Escherichia coli $\mathrm{K} 12$ strain W3110 was the prototrophic parental strain of the gua mutants listed in Table 1.

Bacteriophage. Phage P1 kc was used for transduction as described by Lambden \& Drabble (1973).

Media. Defined salts medium (minimal medium) was that of Vogel \& Bonner (1956), containing glucose (final concentration $2 \mathrm{mg} \mathrm{ml}^{-1}$ ) as carbon source. Minimal agar medium was prepared with $1.5 \%(\mathrm{w} / \mathrm{v})$ Bacto-agar (Difco). Purines, when required, were added to media at a final concentration of $20 \mu \mathrm{g} \mathrm{ml}^{-1}$. Thiamin was used at a final concentration of $5 \mu \mathrm{g} \mathrm{m}^{-1}$. L-G broth, L-G agar (for the propagation of P1kc), nutrient broth and soft agar were prepared as described by Lambden \& Drabble (1973).

Chemicals. Adenosine 5'-triphosphate (disodium salt, trihydrated) and $\beta$-NAD ${ }^{+}$were obtained from Boehringer. Adenine sulphate, glutathione (reduced), guanine hydrochloride and Tris were obtained from Sigma. 6-Chloropurine ribotide (Cl-IMP) was from Calbiochem. Freund's complete adjuvant was from Difco. Ethyl methanesulphonate (EMS) was obtained from Eastman Kodak (Rochester, N.Y., U.S.A.), and $N$-methyl- $N^{\prime}$-nitro- $N$-nitrosoguanidine (NG) from Ralph N. Emanuel (Wembley, HA0 1PY). ICR191E (2-methoxy-6-chloro-9-[3-(2-chloroethyl)aminopropylamine]acridine dihydrochloride) was a gift from Dr H. J. Creech, The Institute for Cancer Research, Philadelphia, Pa, U.S.A. All other chemicals were from BDH.

Isolation of NG-induced mutants. Exponential phase cultures in L-G broth were centrifuged and the cells were resuspended to the same cell density in $0.1 \mathrm{M}$-Tris/maleate buffer, $\mathrm{pH} 6.0$. NG was added $\left(20 \mu \mathrm{g} \mathrm{ml}^{-1}\right.$ final concentration) for $30 \mathrm{~min}$ at $37^{\circ} \mathrm{C}$, then the bacteria were washed in sterile saline $(0.85 \%, \mathrm{w} / \mathrm{v}, \mathrm{NaCl})$ and diluted 50-fold into minimal medium containing guanine $\left(20 \mu \mathrm{g} \mathrm{ml}^{-1}\right)$. This medium allowed growth of the remaining prototrophs and phenotypic expression of guanine auxotrophs. After overnight incubation at $37^{\circ} \mathrm{C}$, the bacteria were washed twice with sterile saline and diluted into minimal medium containing adenine and thiamin to give an absorbance at $610 \mathrm{~nm}$ of about 0.03 . This medium allowed growth of prototrophs and pur mutants, but not gua mutants. After a fourfold increase in cell mass (monitored by the increase in absorbance at $610 \mathrm{~nm}$ ) to allow depletion of guanine pools, benzylpenicillin was added (final concentration 300 units $\mathrm{ml}^{-1}$ ). When lysis was complete (no further decrease in absorbance), the bacteria were centrifuged, resuspended in sterile water, and serial dilutions were made to $10^{-2}$ for replica-plating (Lambden \& Drabble, 1973).

GuaB guaA double mutants were isolated from guaB mutants after NG mutagenesis. Penicillin enrichment of guaB guaA double mutants was made in minimal medium supplemented with xanthine. The double mutants were detected by replica-plating from guanine-supplemented to xanthine-supplemented minimal agar.

For all selections only one mutant of a particular phenotype was retained from each mutagen-treated culture.

Isolation of EMS-induced mutants. The method was described by Lambden \& Drabble (1973).

Mutagen-induced reversion. The methods were described by Lambden \& Drabble (1973), except for reversion induced by 2-aminopurine (2AP): $50 \mu \mathrm{l}$ of a solution containing $1 \mathrm{mg}^{2} \mathrm{AP} \mathrm{ml}^{-1}$ was added to a sterile filter paper disc (13 mm diameter) placed at the centre of the agar plate.

Suppression of amber and ochre mutations. Inocula from overnight cultures of the guaB mutant to be tested and Salmonella typhimurium SL4528 [( colE1) leu malB cysI gal his (amber) $\left(\mathrm{F}^{\prime}-1\right.$-gal ${ }^{+}$sup-812)] (MacPhee \& Stocker, 1969) were mixed in fresh L-G broth and incubated at $37^{\circ} \mathrm{C}$ overnight. The cells were then washed twice with sterile saline and plated on to unsupplemented minimal agar. The plates were incubated for up to $4 \mathrm{~d}$ at $37^{\circ} \mathrm{C}$ before colonies were scored. As a control, the guaB mutant was mixed with $S$. typhimurium SL4257 [( colE1) leu malB cysI gal his (amber) $\left(\mathrm{F}^{\prime}-1-\right.$ gal $\left.\left.^{+}\right)\right]$using the same procedure; no growth was observed on unsupplemented minimal medium.

Enzyme assays. The growth of cultures, preparation of cell-free extracts and the assay for GMP synthetase (EC 6.3 .4 .1) were described by Lambden \& Drabble (1973). IMP dehydrogenase (EC 1 2 .1 14) was assayed according to Gilbert et al. (1979).

Complementation tests. Cell-free extracts were prepared as described by Lambden \& Drabble (1973). Extracts $(2 \mathrm{ml})$ were denatured by adding 2-mercaptoethanol to a final concentration of $0 \cdot 1 \mathrm{~m}$ followed by $1.14 \mathrm{~g}$ solid guanidine hydrochloride over a $5 \mathrm{~min}$ period. The extracts were then left at $4{ }^{\circ} \mathrm{C}$ for $30 \mathrm{~min}$. Denatured extracts from two different guaB mutants were mixed and then dialysed twice over a $16 \mathrm{~h}$ period

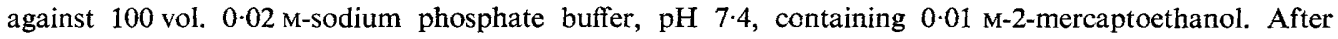
removing precipitated protein by centrifugation, the supernatant was assayed for IMP dehydrogenase activity.

Covalent modification of IMP dehydrogenase from guaB mutants. Cell-free extracts $(1 \mathrm{ml}$, containing $5 \mathrm{mM}$ 
Table 1. Growth patterns of pur and gua mutants

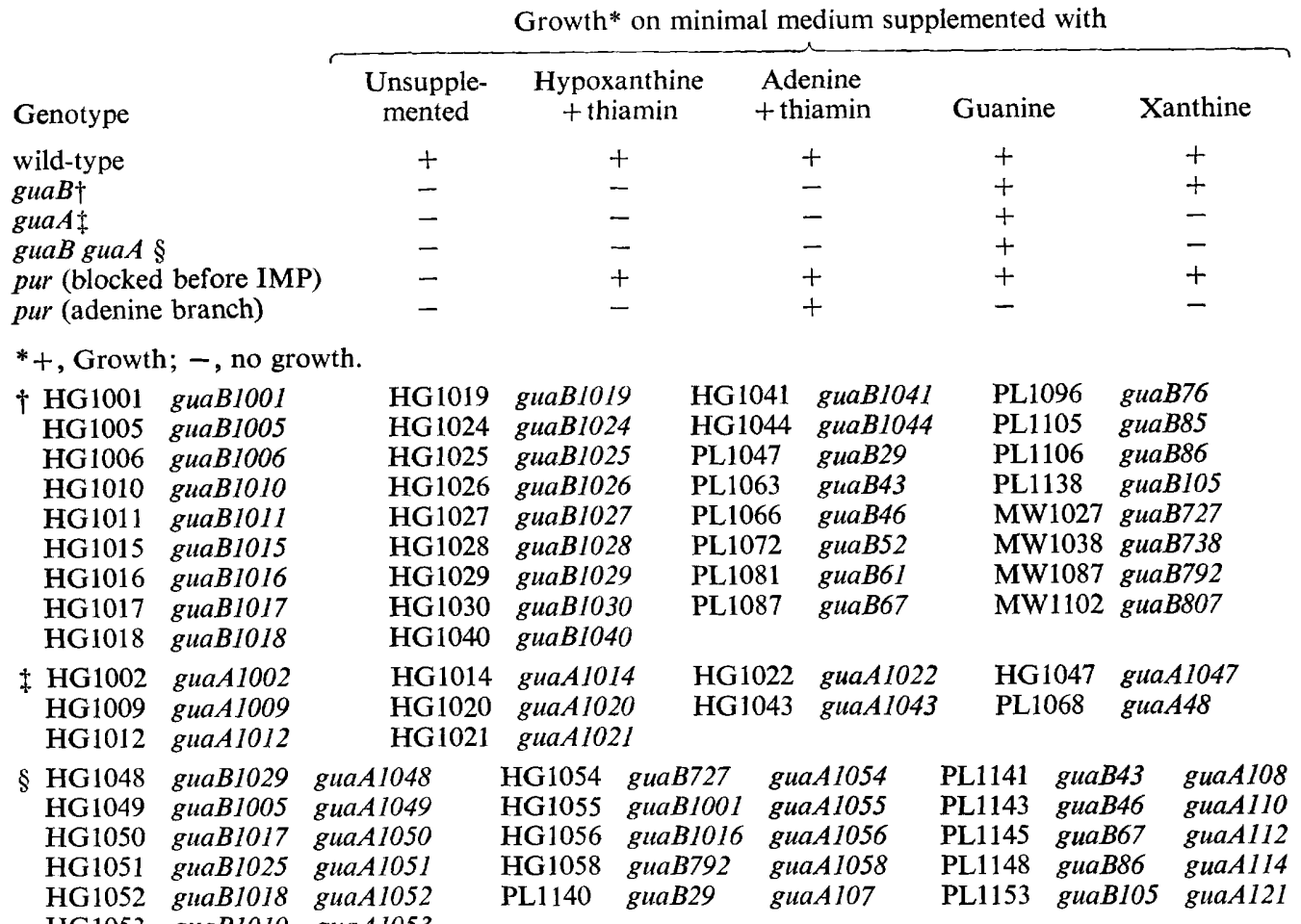

reduced glutathione) of guaB mutants and the guaA mutant PL1068 were treated with $100 \mathrm{nmol}$ chloropurine ribotide (Cl-IMP) and the resultant loss of IMP dehydrogenase activity was followed for the guaA mutant. When total inactivation of the native enzyme had occurred (approximately 2 min) all the extracts were left for a further $30 \mathrm{~min}$ to ensure complete reaction of the mutant enzymes with Cl-IMP. IMP was then added to a concentration of $2 \mathrm{~mm}$. The preparations were dialysed three times for $8 \mathrm{~h}$ against $100 \mathrm{vol}$. $50 \mathrm{~mm}$-potassium phosphate buffer, $\mathrm{pH} 7 \cdot 4$, containing $1 \mathrm{mM}$ reduced glutathione and $2 \mathrm{mM}$-IMP. Mutant extracts (treated with Cl-IMP and untreated) were then denatured with guanidine hydrochloride $(5 \mathrm{mM}$ reduced glutathione replacing 2-mercaptoethanol) and mixed such that for each pair of mutants four types of hybrid enzyme were produced - covalently modified subunits from one or other of the mutants, all subunits modified or no subunit modified. IMP was present throughout to protect the hybrid enzymes from any Cl-IMP which had not been removed by prior dialysis. After renaturation, IMP dehydrogenase activity was determined for the various hybrid enzymes produced. Similar treatment was given to enzymes purified from HG1005 and PL1138 (purification descrited by Gilbert et al., 1979). Covalent modification was achieved by adding $8 \mathrm{nmol}$ portions of Cl-IMP until the reaction was complete (indicated by the absorbance at $290 \mathrm{~nm}$ reaching a maximum value). The enzymes were dialysed, denatured, mixed and renatured as described above, and finally assayed for IMP dehydrogenase activity.

Immunological methods. These were described by Gilbert et al. (1979).

\section{RESULTS}

\section{Characterization of mutants}

Preliminary classification of gua mutants (Table 1) was based on their growth on media supplemented with various purines. GuaA mutants have a specific requirement for guanine, but guaB mutants respond to guanine and xanthine. GuaB guaA double mutants have the same growth characteristics as guaA mutants.

The activities of IMP dehydrogenase and GMP synthetase were determined to confirm 
H. J. GILBERT AND W. T. DRABBLE

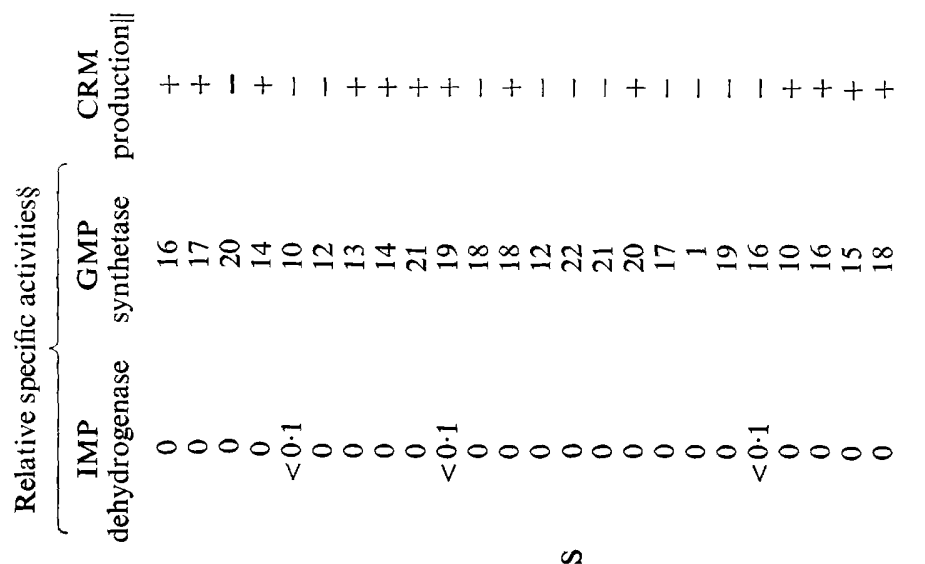

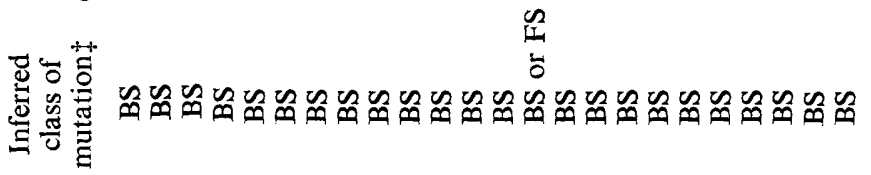

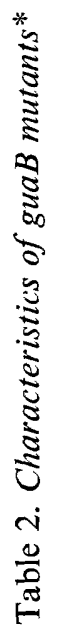

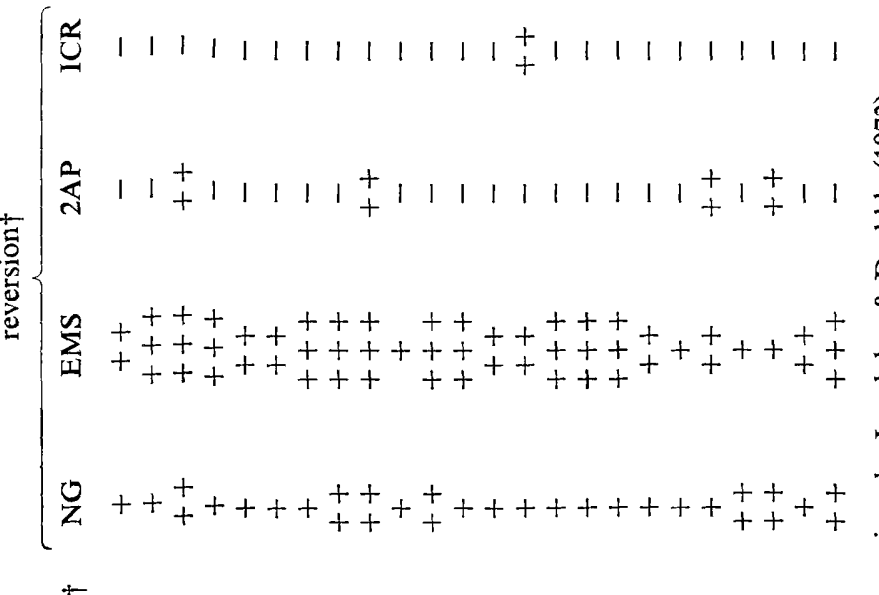

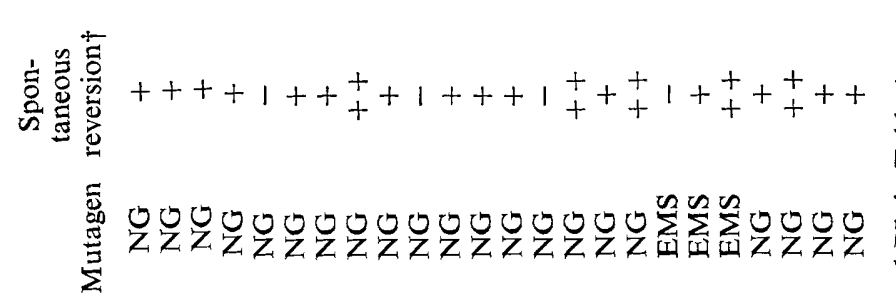

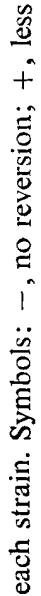

$\stackrel{i}{\Xi}$

$\sum_{0}$

ఏ

응

$\cong$

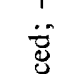

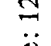

焉

䓃

离

$+$

童

o

焉

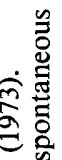

능

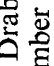

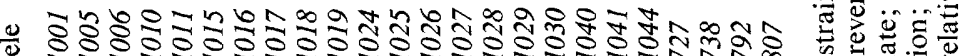

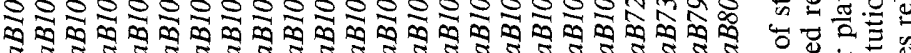

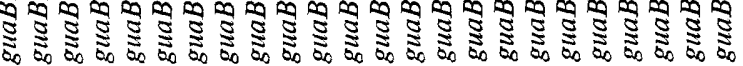




\section{Table 3. Suppression of guaB mutations}

The procedure used is described in Methods.

guaB strains tested

PL1063 (guaB43)*

PL1081 (guaB6l)*

PL1047 (guaB29)*
Response

Growth after $2 \mathrm{~d}$

Growth after $3-4 \mathrm{~d}$

No growth after $4 \mathrm{~d}$

HG1001, HG1005, HG1006, HG1010, HG1011, HG1015,

HG1016, HG1017, HG1018, HG1019, HG1024, HG1025,

HG1026, HG1027, HG1028, HG1029, HG1030, HG1040,

No growth after $4 \mathrm{~d}$

* These strains carry base substitution polar mutations (Lambden \& Drabble, 1973).

the preliminary classification of gua mutants (Table 2). All guaB mutants were shown to lack a functional IMP dehydrogenase and produced, except for one mutant, derepressed levels of GMP synthetase. The exceptional mutant (HG1040) probably carries a polar mutation in guaB which exerts an effect on the adjacent guaA gene leading to low GMP synthetase activity. Polar guaB mutants of this type have been described previously by Lambden \& Drabble (1973). Strains with a growth response characteristic of guaA mutations produced derepressed levels of IMP dehydrogenase but in no case was GMP synthetase detected (results not shown). No guaB guaA double mutant showed IMP dehydrogenase or GMP synthetase activity when grown under conditions for derepression.

The presence of protein (CRM) cross-reacting with anti-(IMP dehydrogenase) antibodies was detected by double immuno-gel diffusion (Table 2). The use of two monospecific antisera to IMP dehydrogenase, prepared by different methods (Gilbert et al., 1979), gave identical results. Of the 24 mutants tested, 13 were CRM ${ }^{+}$. However, HG1001 and HG1017 gave fainter precipitin lines than other $\mathrm{CRM}^{+}$strains. The mutant enzyme in these strains may be particularly susceptible to proteolytic degradation so that relatively low concentrations of the protein would be present in cell-free extracts. Spurs were observed between extracts of HG1029 and PL1068 (guaA48) suggesting that not all the antigenic sites of native IMP dehydrogenase are present on the protein produced by HG1029. All other cellfree extracts from guaB mutants produced complete fusion of precipitin lines with extracts containing native IMP dehydrogenase indicating antigenic identity of the mutant and native enzymes.

$G u a B$ mutants were further classified according to their mutagen-induced reversion patterns (Table 2). The mutations appear to be of the base substitution type with the possible exception of guaB1027 which may be a frameshift mutation. 2-Aminopurine causes transitions between AT and GC base pairs. Therefore the mutations which are reverted by 2-aminopurine have AT replacing the original GC as both NG and EMS cause a GC to AT exchange.

Suppression of guaB mutations was determined by conjugational transfer of an $\mathrm{F}^{\prime}$ factor carrying an ochre suppressor gene (MacPhee \& Stocker, 1969) from S. typhimurium to a series of guaB mutant strains (Table 3). Three strains with known base-substitution polar mutations [PL1047 (guaB29), PL1063 (guaB43) and PL1081 (guaB61)] isolated by Lambden $\&$ Drabble (1973) were included in this test to act as controls. Of these three polar mutations, guaB43 and guaB61 were suppressed while guaB29 was not. PL1081 (guaB61) grew rather slowly. This suggests that guaB43 is an ochre mutation whereas guaB61 is an amber mutation because ochre suppressors are more effective against ochre than against amber mutations. GuaB29 presumably generates a UGA termination codon as the ochre suppressor has no effect on this type of mutation. No other guaB strain tested responded to the suppressor suggesting that none carries amber or ochre mutations. The polar mutant HG1040 presumably has a UGA mutation. 


\section{Table 4. Data from reciprocal three-factor crosses}

Reciprocal crosses were performed as described by Lambden \& Drabble (1973). Wild-type recombinants $\left(\mathrm{guaB}^{+} \mathrm{guaA^{+ }}\right)$ were selected on unsupplemented minimal medium; guaA $A^{+}$recombinants ( $g u a B^{+} g u a A^{+}$and $\left.g u a B g u a A^{+}\right)$were selected on xanthine-supplemented medium.

\begin{tabular}{|c|c|c|c|c|c|c|}
\hline \multirow{2}{*}{$\begin{array}{c}\text { Donor } \\
\text { allele }\end{array}$} & \multirow{2}{*}{\multicolumn{2}{|c|}{ Recipient alleles }} & \multicolumn{2}{|c|}{$\begin{array}{c}\text { Transductants } \\
\text { selected }\left(10^{6} \times \text { f.o.t. }\right)^{*}\end{array}$} & \multirow{2}{*}{$\frac{B^{+} A^{+}}{A^{+}}$} & \multirow{3}{*}{ Inferred order } \\
\hline & & & & & & \\
\hline guaB1001 & guaBl00I & guaA1055 & 0.0000 & $2 \cdot 40$ & $\begin{array}{c}(\%) \\
0.00\end{array}$ & \\
\hline guaB1005 & guaB1005 & guaA 1049 & 0.0000 & $3 \cdot 40$ & 0.00 & \\
\hline$g u a B 1016$ & guaB 1016 & guaA 1056 & 0.0000 & $1 \cdot 30$ & 0.00 & \\
\hline guaB1017 & guaB 1017 & guaA1050 & 0.0000 & $5 \cdot 20$ & 0.00 & \\
\hline guaBlo18 & guaB1018 & guaA1052 & 0.0000 & $4 \cdot 60$ & 0.00 & \\
\hline guaB792 & guaB792 & guaA1058 & 0.0000 & $6 \cdot 70$ & $0 \cdot 00$ & \\
\hline guaB29 & guaB29 & guaA 107 & 0.0000 & 0.80 & 0.00 & \\
\hline guaB43 & guaB43 & guaA 108 & 0.0000 & 0.87 & 0.00 & \\
\hline guaB67 & guaB67 & guaA112 & 0.0000 & $1 \cdot 20$ & 0.00 & \\
\hline guaB86 & guaB86 & guaA114 & 0.0000 & $5 \cdot 20$ & 0.00 & \\
\hline guaBI0s & guaB105 & guaA121 & $0 \cdot 0000$ & $2 \cdot 50$ & 0.00 & \\
\hline guaB46 & guaB46 & guaA110 & 0.0000 & $3 \cdot 20$ & 0.00 & \\
\hline guaB1001 & guaB105 & guaA121 & 0.0140 & 0.42 & $3 \cdot 33$ & \\
\hline guaB105 & guaB1001 & guaA1055 & 0.0070 & 0.82 & 0.85 & B1001-B105-guaA \\
\hline guaBlo05 & guaB105 & guaA121 & 0.0610 & 0.68 & $8 \cdot 97$ & \\
\hline guaB105 & guaB1005 & guaA1049 & 0.0008 & 0.48 & $0 \cdot 17$ & B1005-B105-guaA \\
\hline guaBI016 & guaB105 & guaA121 & 0.0060 & $0 \cdot 12$ & $5 \cdot 00$ & \\
\hline guaBlo5 & guaB1016 & guaA1056 & 0.0088 & $2 \cdot 40$ & 0.37 & B1016-B105-guaA \\
\hline guaB1018 & guaB105 & guaA121 & 0.0800 & $3 \cdot 50$ & $2 \cdot 29$ & \\
\hline guaB105 & guaB1018 & guaA1052 & 0.0008 & $0 \cdot 31$ & 0.26 & B1018-B105-guaA \\
\hline guaB792 & guaB105 & guaA121 & 0.0800 & $7 \cdot 07$ & $1 \cdot 13$ & \\
\hline guaBI05 & guaB792 & guaA1058 & $0 \cdot 0065$ & 0.93 & $0 \cdot 70$ & B792-B105-guaA \\
\hline guaB29 & guaB105 & guaA121 & 0.0091 & 0.89 & $1 \cdot 02$ & \\
\hline guaBlo5 & guaB29 & guaA107 & 0.0061 & $2 \cdot 13$ & $0 \cdot 29$ & B29-B105-guaA \\
\hline guaB46 & guaB 105 & guaA121 & 0.0550 & $3 \cdot 31$ & 1.66 & \\
\hline guaB105 & guaB46 & guaA110 & 0.0400 & $11 \cdot 12$ & $0 \cdot 36$ & B46-B105-guaA \\
\hline guaB67 & guaB 105 & guaA121 & $0 \cdot 0400$ & $0 \cdot 60$ & $6 \cdot 67$ & \\
\hline guaBlo5 & guaB67 & guaA112 & $0 \cdot 0234$ & $2 \cdot 09$ & $1 \cdot 12$ & B67-B105-guaA \\
\hline guaB86 & guaB105 & guaA121 & 0.0102 & $1 \cdot 76$ & 0.58 & \\
\hline guaB105 & guaB86 & guaA114 & $0 \cdot 1320$ & $9 \cdot 76$ & $1 \cdot 36$ & B105-B86-guaA \\
\hline guaB1018 & guaB46 & guaA110 & 0.0448 & $5 \cdot 60$ & $0 \cdot 80$ & \\
\hline guaB46 & guaB1018 & guaA1052 & $0 \cdot 0030$ & $2 \cdot 30$ & $0 \cdot 13$ & B1018-B46-guaA \\
\hline guaB1018 & guaB1005 & guaA1049 & 0.0004 & $4 \cdot 24$ & 0.01 & \\
\hline guaB1005 & guaB1018 & guaA1052 & $0 \cdot 0130$ & $0 \cdot 30$ & $4 \cdot 33$ & B1005-B1018-guaA \\
\hline guaB1018 & guaB1017 & guaAI050 & 0.0750 & $10 \cdot 00$ & 0.75 & \\
\hline guaB1017 & guaB1018 & guaA1052 & $0 \cdot 0030$ & 0.48 & 0.63 & B1018-B1017-guaA \\
\hline guaB1018 & guaB43 & guaA108 & 0.0006 & $2 \cdot 00$ & 0.03 & \\
\hline guaB43 & guaB1018 & guaA1052 & $0 \cdot 0040$ & $0 \cdot 26$ & $1 \cdot 54$ & B43-B1018-guaA \\
\hline guaB1018 & guaB29 & guaA107 & 0.0827 & $5 \cdot 30$ & 1.56 & \\
\hline guaB29 & guaB1018 & guaA1052 & 0.0050 & $0 \cdot 70$ & $0 \cdot 71$ & B1018-B29-guaA \\
\hline guaB 1017 & guaB43 & guaA108 & 0.0047 & 4.90 & $0 \cdot 10$ & \\
\hline guaB43 & guaB1017 & guaA1050 & 0.0041 & $0 \cdot 32$ & $1 \cdot 28$ & B43-B1017-guaA \\
\hline guaB1017 & guaB67 & guaA112 & 0.0052 & $3 \cdot 50$ & $0 \cdot 15$ & \\
\hline guaB67 & guaB1017 & guaA1050 & 0.0064 & $0 \cdot 13$ & $4 \cdot 92$ & B67-B1017-guaA \\
\hline guaB1017 & guaB1001 & guaA1055 & 0.0020 & $0 \cdot 23$ & 0.87 & \\
\hline guaBlo01 & guaB1017 & guaA1050 & 0.0026 & $0 \cdot 10$ & $2 \cdot 60$ & B1001-B1017-guaA \\
\hline guaB1016 & guaB67 & guaA112 & 0.0622 & $6 \cdot 00$ & $1 \cdot 04$ & \\
\hline guaB67 & guaB1016 & guaA1056 & 0.0100 & 0.57 & $1 \cdot 75$ & B67-B1016-guaA \\
\hline guaB1005 & guaB67 & guaAl12 & $0 \cdot 0340$ & $1 \cdot 60$ & $2 \cdot 13$ & \\
\hline guaB67 & guaB1005 & guaA1049 & 0.0100 & $1 \cdot 00$ & $1 \cdot 00$ & B1005-B67-guaA \\
\hline
\end{tabular}


Table 4. (cont.)

\begin{tabular}{|c|c|c|c|c|c|c|}
\hline \multirow{3}{*}{$\begin{array}{l}\begin{array}{c}\text { Donor } \\
\text { allele }\end{array} \\
\text { guaB1001 } \\
\text { guaB792 }\end{array}$} & & & \multicolumn{2}{|c|}{$\begin{array}{c}\text { Transductants } \\
\text { selected }\left(10^{6} \times \text { f.o.t. }\right)^{*}\end{array}$} & \multirow{3}{*}{$\begin{array}{c}\frac{B^{+} A^{+}}{A^{+}} \\
(\%) \\
2 \cdot 14 \\
0.94\end{array}$} & \multirow{3}{*}{ Inferred order } \\
\hline & \multicolumn{2}{|c|}{ Recipient alleles } & gua $B^{+} g u a A^{+}$ & $\mathrm{guaA}^{+}$ & & \\
\hline & $\begin{array}{l}\text { guaB792 } \\
\text { guaB1001 }\end{array}$ & $\begin{array}{l}\text { guaA } 107 \\
\text { guaA } 1055\end{array}$ & $\begin{array}{l}0 \cdot 0150 \\
0 \cdot 0150\end{array}$ & $\begin{array}{l}0.70 \\
1.60\end{array}$ & & \\
\hline $\begin{array}{l}\text { guaB792 } \\
\text { guaB86 }\end{array}$ & $\begin{array}{l}\text { guaB86 } \\
\text { guaB792 }\end{array}$ & $\begin{array}{l}\text { guaA114 } \\
\text { guaA } 1058\end{array}$ & $\begin{array}{l}0.0120 \\
0.0500\end{array}$ & $\begin{array}{l}0 \cdot 62 \\
7 \cdot 20\end{array}$ & $\begin{array}{l}1.93 \\
0.69\end{array}$ & B792-B86-guaA \\
\hline $\begin{array}{l}\text { guaB46 } \\
\text { guaB86 }\end{array}$ & $\begin{array}{l}\text { guaB86 } \\
\text { guaB46 }\end{array}$ & $\begin{array}{l}\text { guaA114 } \\
\text { guaA110 }\end{array}$ & $\begin{array}{l}0.0030 \\
0 \cdot 0020\end{array}$ & $\begin{array}{l}0 \cdot 11 \\
1 \cdot 20\end{array}$ & $\begin{array}{l}2 \cdot 72 \\
0 \cdot 17\end{array}$ & B46-B86-guaA \\
\hline $\begin{array}{l}\text { guaB46 } \\
\text { guaB29 }\end{array}$ & $\begin{array}{l}\text { guaB29 } \\
\text { guaB46 }\end{array}$ & $\begin{array}{l}\text { guaA107 } \\
\text { guaA110 }\end{array}$ & $\begin{array}{l}0.0016 \\
0.0014\end{array}$ & $\begin{array}{l}0 \cdot 12 \\
0 \cdot 23\end{array}$ & $\begin{array}{l}1 \cdot 33 \\
0 \cdot 61\end{array}$ & B46-B29-guaA \\
\hline $\begin{array}{l}\text { guaB1005 } \\
\text { guaB46 }\end{array}$ & $\begin{array}{l}\text { guaB46 } \\
\text { guaB1005 }\end{array}$ & $\begin{array}{l}\text { guaA110 } \\
\text { guaA1049 }\end{array}$ & $\begin{array}{l}0.0840 \\
0.0010\end{array}$ & $\begin{array}{l}1 \cdot 62 \\
1 \cdot 00\end{array}$ & $\begin{array}{l}5 \cdot 18 \\
0 \cdot 10\end{array}$ & B1005-B46-guaA \\
\hline $\begin{array}{l}\text { guaB } 1017 \\
\text { guaB } 105\end{array}$ & $\begin{array}{l}\text { guaB105 } \\
\text { guaB1017 }\end{array}$ & $\begin{array}{l}\text { guaA121 } \\
\text { guaA } 1050\end{array}$ & $\begin{array}{l}0.0748 \\
0.0270\end{array}$ & $\begin{array}{l}4 \cdot 09 \\
2 \cdot 37\end{array}$ & $\begin{array}{l}1 \cdot 82 \\
1 \cdot 14\end{array}$ & B1017-B105-guaA \\
\hline
\end{tabular}

* f.o.t. (frequency of transduction) is the number of transductants obtained per phage added.

\section{Mapping of guaB mutations by three-factor crosses}

Mutations were mapped at the guaB locus by transductional three-factor crosses using guaA as the outside reference marker. The principle of the cross was described by Lambden $\&$ Drabble (1973). The recipient strain in the crosses carried an additional mutation in the closely linked guaA gene. Pairs of guaB mutations were thus ordered with respect to the third reference guaA mutation. Wild-type recombinants ( $\left.g u a B^{+} g u a A^{+}\right)$were selected on unsupplemented minimal medium whereas $g u a A^{+}$recombinants (guaB ${ }^{+} \mathrm{guaA}^{+}$and guaB gua $A^{+}$) were selected on xanthine-supplemented medium. Wild-type recombinants were expressed as a percentage of the total $\mathrm{guaA}^{+}$recombinants (Table 4).

The order of guaB mutations with respect to the guaA marker can be deduced from the recombination frequencies for a particular pair of reciprocal crosses. The higher value of $\mathrm{B}^{+} \mathrm{A}^{+} / \mathrm{A}^{+}$was taken as an indication of the distance between guaB mutations (Fig. 1). The map (Fig. 1) also incorporates data of Lambden \& Drabble (1973). A major feature of the map is a clustering of mutations in the operator distal region delineated by guaB1001 and guaB105. This may indicate an important role for the amino acid sequence coded by this region in the correct formation of enzyme tertiary structure. There is no consistent pattern of mutations giving rise to CRM.

\section{Complementation in vitro between guaB mutants}

No IMP dehydrogenase activity was observed when cell-free extracts of guaB mutants were individually denatured with guanidine hydrochloride and renatured, nor when they were mixed in the absence of denaturing conditions. Mixing the extract of any $\mathrm{CRM}^{+}$ mutant with that of PL1138 (guaB105) in denaturing conditions resulted in restoration of enzyme activity after renaturation (Table 5). The activity observed depended on the particular $\mathrm{CRM}^{+}$mutant used to complement PL1138. The combination of extracts from PL1138 and HG1005 produced the most active enzyme. Extracts of CRM- mutants, when mixed with an extract of PL1138, produced no enzyme activity. No other combination of extracts resulted in complementation.

\section{Heat stability of native and hybrid forms of IMP dehydrogenase}

The heat stability of native enzyme, reconstituted native enzyme and two hybrid enzymes (PL1138-HG1001 and PL1138-HG1005 extract combinations) was examined (Fig. 2). Under the conditions used both native and reconstituted IMP dehydrogenases were stable 


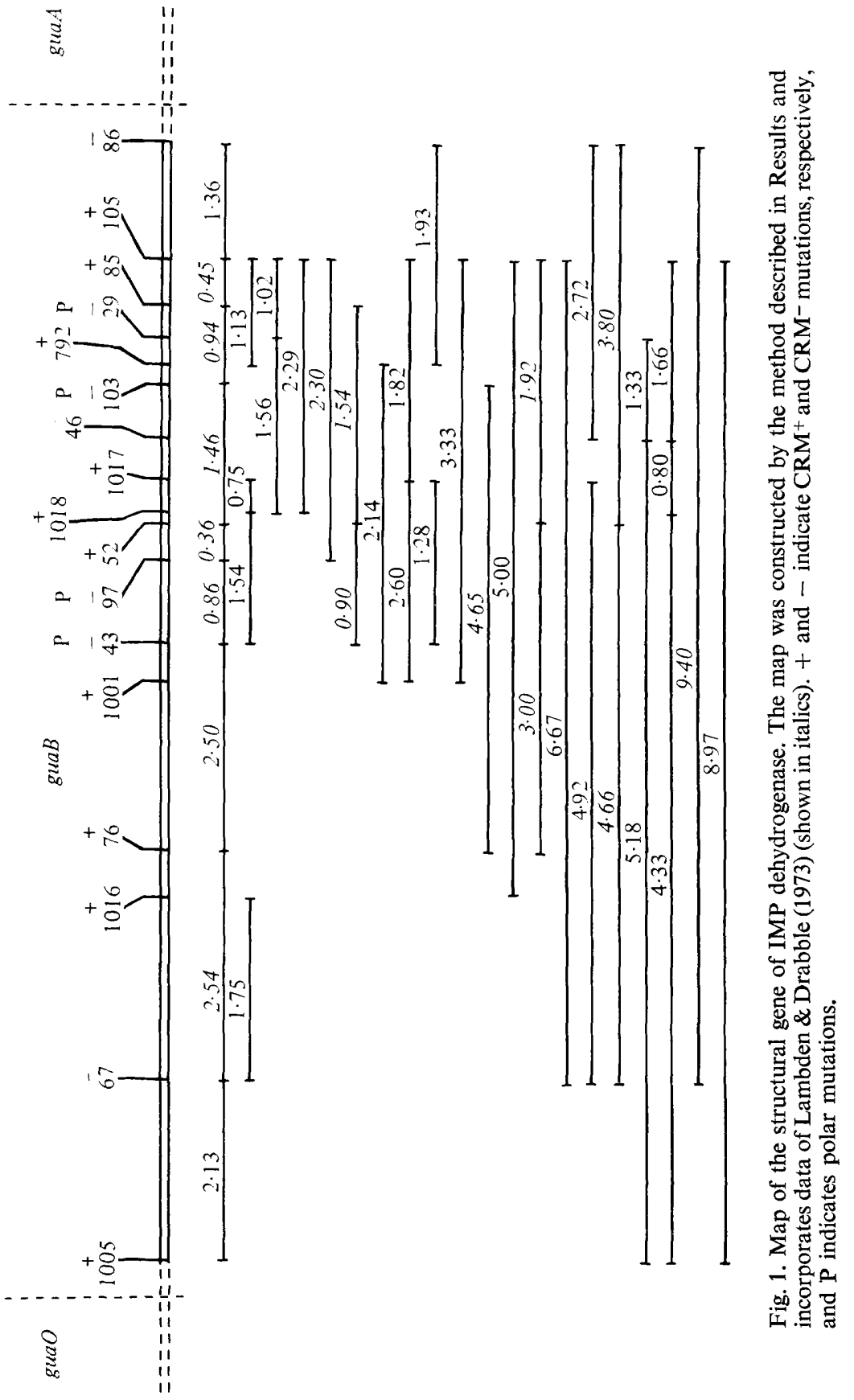


Table 5. Complementation in vitro between guaB mutants

Complementation was performed as described in Methods. The results show the specific activities of IMP dehydrogenase $(\%)$ compared with denatured and renatured native enzyme (a cell-free extract of derepressed PL1068) which has a specific activity of $0.3 \mu \mathrm{mol} \mathrm{XMP} \mathrm{min}^{-1}\left(\mathrm{mg}^{2}\right.$ protein) ${ }^{-1}$ $(=100 \%)$.

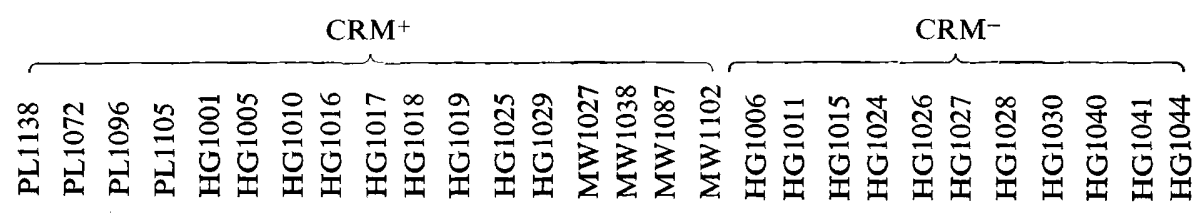

CRM $^{+}\left(\begin{array}{l}\text { PL1138 } \\ \text { PL1072 } \\ \text { PL1096 } \\ \text { PL1105 } \\ \text { HG1001 } \\ \text { HG1005 } \\ \text { HG1010 } \\ \text { HG1016 } \\ \text { HG1017 } \\ \text { HG1018 } \\ \text { HG1019 } \\ \text { HG1025 } \\ \text { HG1029 } \\ \text { MW1027 } \\ \text { MW1038 } \\ \text { MW1087 } \\ \text { MW1102 } \\ \text { HG1006 } \\ \text { HG1011 } \\ \text { HG1015 } \\ \text { HG1024 } \\ \text { HG1026 } \\ \text { HG1027 } \\ \text { HG1028 } \\ \text { HG1030 } \\ \text { HG1040 } \\ \text { HG1041 } \\ \text { HG1044 }\end{array}\right.$

$$
0
$$$$
270
$$$$
\begin{array}{lll}
23 & 0 & 0
\end{array}
$$$$
30 \quad 00000
$$$$
\begin{array}{lllll}
9 & 0 & 0 & 0 & 0
\end{array}
$$$$
\begin{array}{llllll}
35 & 0 & 0 & 0 & 0 & 0
\end{array}
$$$$
\begin{array}{lllllll}
17 & 0 & 0 & 0 & 0 & 0 & 0
\end{array}
$$

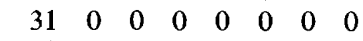$$
\begin{array}{lllllllll}
13 & 0 & 0 & 0 & 0 & 0 & 0 & 0 & 0
\end{array}
$$$$
\begin{array}{llllllllll}
27 & 0 & 0 & 0 & 0 & 0 & 0 & 0 & 0 & 0
\end{array}
$$$$
\begin{array}{lllllllllll}
15 & 0 & 0 & 0 & 0 & 0 & 0 & 0 & 0 & 0 & 0
\end{array}
$$$$
\begin{array}{llllllllllll}
33 & 0 & 0 & 0 & 0 & 0 & 0 & 0 & 0 & 0 & 0 & 0
\end{array}
$$$$
\begin{array}{llllllllllllll}
28 & 0 & 0 & 0 & 0 & 0 & 0 & 0 & 0 & 0 & 0 & 0 & 0
\end{array}
$$$$
\begin{array}{lllllllllllllll}
21 & 0 & 0 & 0 & 0 & 0 & 0 & 0 & 0 & 0 & 0 & 0 & 0 & 0
\end{array}
$$$$
\begin{array}{llllllllllllllll}
25 & 0 & 0 & 0 & 0 & 0 & 0 & 0 & 0 & 0 & 0 & 0 & 0 & 0 & 0
\end{array}
$$$$
\begin{array}{llllllllllllllllll}
16 & 0 & 0 & 0 & 0 & 0 & 0 & 0 & 0 & 0 & 0 & 0 & 0 & 0 & 0 & 0 & \\
19 & 0 & 0 & 0 & 0 & 0 & 0 & 0 & 0 & 0 & 0 & 0 & 0 & 0 & 0 & 0 & 0
\end{array}
$$$$
\begin{array}{lrrrrrrrrrrrrrrrrrr}
\text { MW1102 } & 19 & 0 & 0 & 0 & 0 & 0 & 0 & 0 & 0 & 0 & 0 & 0 & 0 & 0 & 0 & 0 & 0 \\
\text { HG1006 } & 0 & - & - & - & - & - & - & - & - & - & - & - & - & - & - & - & - & 0
\end{array}
$$$$
\text { HG1011 } 0 \text { - }- \text { - }-0 \text { - } 0 \text { - } 00-0 \text { - } 0 \text { - }-0
$$

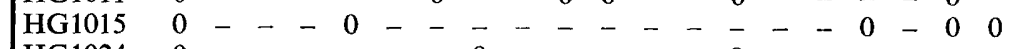$$
\text { HG1024 } 0 \text { - }- \text { - }- \text { - } 0-- \text { - }-0-\text { - }- \text { - } 0
$$

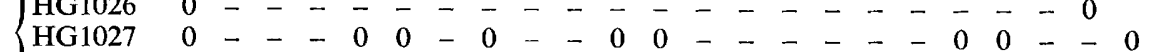

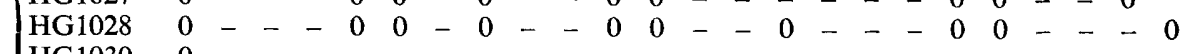

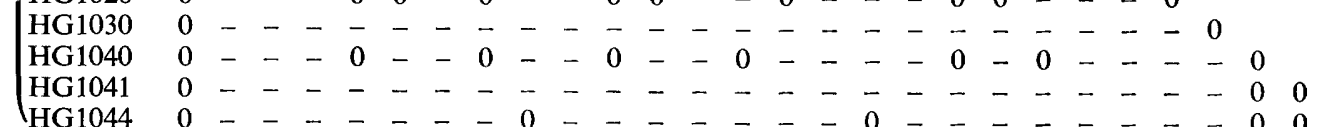

$$
-, \text { Not tested. }
$$

for up to $30 \mathrm{~min}$ at $70^{\circ} \mathrm{C}$ and both had the same susceptibility to heat inactivation at higher temperatures (Fig. 2). This suggests that the tertiary structure of the enzyme is unchanged by denaturation and renaturation. The hybrid enzymes were more heat labile. The more enzymically active hybrid (PL1138-HG1005) showed the greater heat stability. Loss of enzyme activity at each temperature was first order with respect to time (Fig. $2 b$ ).

\section{Kinetic properties of native and hybrid IMP dehydrogenases}

Native and hybrid (PL1138-HG1001 and PL1138-HG1005) IMP dehydrogenases were purified as described by Gilbert et al. (1979). The kinetic constants (Table 6) of these enzymes were determined at a fixed concentration of NAD for the $K_{\mathrm{m}}^{\mathrm{IMP}}$ and vice versa for $K_{\mathrm{m}}^{\mathrm{NAD}}$. The $K_{\mathrm{i}}^{\mathrm{GMP}}$ was determined at $0 \cdot 1$ and $0.2 \mathrm{mM-GMP}$. The kinetic constants for native, reconstituted native and the hybrid enzymes were similar; therefore the low specific activities of the hybrid enzymes (Table 5) are not related to an unusually high $\mathrm{K}_{\mathrm{m}}$ for one or both of the substrates. 

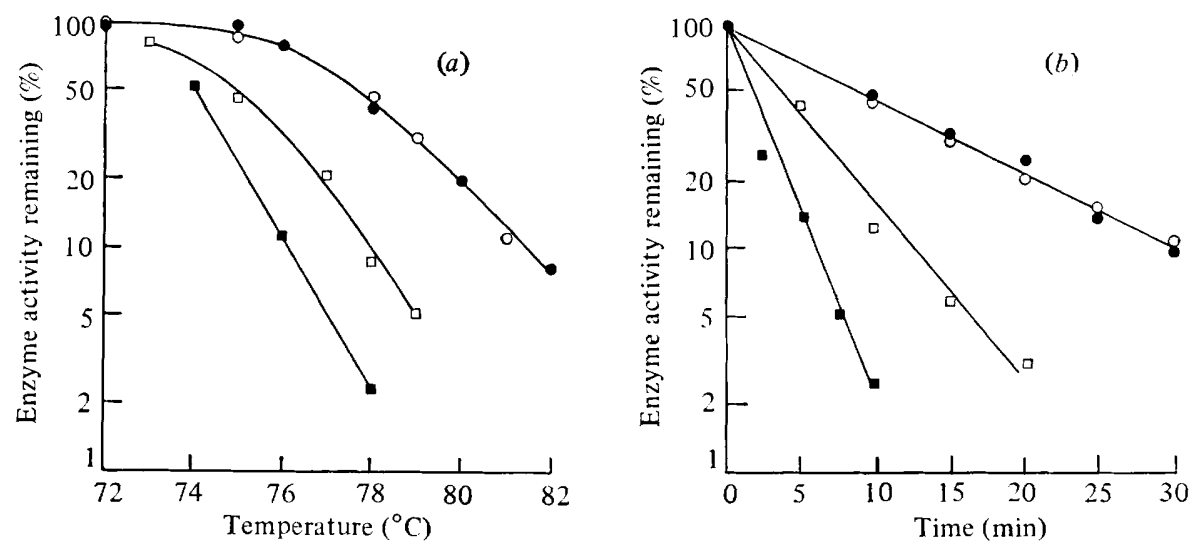

Fig. 2. Heat stability of native and hybrid forms of IMP dehydrogenase. Cell-free extracts were prepared, denatured and renatured as described in Methods. Samples $\left(2 \mathrm{ml}, 5 \mathrm{mg}\right.$ protein $\left.\mathrm{ml}^{-1}\right)$ were incubated either for $10 \mathrm{~min}$ at various temperatures $(a)$ or for various times at $78{ }^{\circ} \mathrm{C}(b)$. Portions $(0.3 \mathrm{ml})$ were withdrawn at the end of the incubation, cooled to $0{ }^{\circ} \mathrm{C}$ and assayed for IMP dehydrogenase activity. $\bigcirc$, Native enzyme present in a cell-free extract from PL1068 (guaA48); ○, reconstituted native enzyme from PL1068 ( $\mathrm{guaA48}$ ); $\square$, hybrid enzyme from mixed extracts of PL1138 and HG1005; $\boldsymbol{\square}$, hybrid enzyme from mixed extracts of PL1138 and HG1001.

\section{Table 6. Kinetic constants for native and hybrid IMP dehydrogenases}

The reaction mixtures contained, in $1 \mathrm{ml}$ : Tris $/ \mathrm{HCl}(\mathrm{pH} \mathrm{7.85}), 50 \mu \mathrm{mol} ; \mathrm{KCl}, 33 \mu \mathrm{mol}$; reduced glutathione, $5 \mu \mathrm{mol}$; and (for the determination of $K_{\mathrm{m}}^{\mathrm{IMP}}$ ) IMP, 0.01 to $0.20 \mu \mathrm{mol}$, and NAD, $0.20,0.40$ or $1.25 \mu \mathrm{mol}$, or (for the determination of $K_{\mathrm{m}}^{\mathrm{NAD}}$ ) NAD, 0.1 to $2.0 \mu \mathrm{mol}$, and IMP, $0.02,0.10$ or $0.20 \mu \mathrm{mol}$. $K_{\mathrm{i}}^{\mathrm{GMP}}$ was determined using 0.10 and $0.20 \mu \mathrm{mol}$ GMP. The results show the kinetic constants \pm the standard error of the mean, with the number of determinations in parentheses.

Enzyme
Native
Denatured and renatured
native
HG1005-PL1138 hybrid
HG1001-PL1138 hybrid

\begin{tabular}{|c|c|c|}
\hline \multicolumn{3}{|c|}{ Kinetic constants $(\mu \mathrm{M})$} \\
\hline$K_{\mathrm{m}}^{\mathrm{IMPP}}$ & $K_{\mathrm{m}}^{\mathrm{NAD}}$ & $K_{\mathrm{i}}^{\mathrm{GMP}}$ \\
\hline $11 \cdot 1 \pm 0 \cdot 6(6)$ & $330 \pm 10(4)$ & $56 \pm 3$ \\
\hline $11 \cdot 3 \pm 0 \cdot 12(3)$ & $341 \pm 26(3)$ & $63 \pm 16(3)$ \\
\hline $10 \cdot 1 \pm 0 \cdot 12$ & $252 \pm 21$ & $56 \pm 8(3)$ \\
\hline $7 \cdot 5 \pm 0 \cdot 15(3)$ & $202 \pm 23(3)$ & $60 \pm 11(3)$ \\
\hline
\end{tabular}

\section{Covalent modification of hybrid IMP dehydrogenase}

Native IMP dehydrogenase and the enzyme from several of the $\mathrm{CRM}^{+}$guaB mutants can be covalently modified at the active site by Cl-IMP (Gilbert \& Drabble, 1978). Thus, the absorption spectrum of Cl-IMP undergoes a characteristic change on incubation of the reagent with purified native or mutant enzymes and the native enzyme becomes inactive. Specific modification at the IMP binding site of the enzyme is suggested by the considerable retardation of this reaction by IMP, and by the non-reactivity of chloro-inosine. Amongst the mutant enzymes modified by Cl-IMP were those produced by HG1005, HG1019, PL1072, PL1105 and PL1138.

Enzyme activity was determined for hybrid enzymes containing subunits which had been covalently modified with Cl-IMP (Table 7). Enzyme activity was absent when either all subunits had been modified or only those subunits derived from PL1138. When subunits from PL1138 had not been modified enzyme activity (75 to $90 \%$ of that of the untreated hybrid enzyme) was observed. These results suggest that in hybrid enzymes the subunits derived from PL1138 are responsible for the catalytic reaction. The complementary subunit may be holding subunits from PL1138 in the correct conformation for catalysis. The reduced activity of the hybrid enzymes with modification to subunits other than those of PL1138 may be a consequence of incomplete removal of Cl-IMP during dialysis. 


\title{
Table 7. Covalent modification of hybrid IMP dehydrogenase by Cl-IMP
}

\begin{abstract}
The experiments were performed as described in Methods. Purified enzyme (Gilbert et al., 1979) was used in the first experiment listed; in all others, hybrid enzyme was formed from cell-free extracts. In each case, the enzyme activity of the modified enzyme is shown relative to that of the unmodified control preparation $(=100 \%)$.
\end{abstract}

$\begin{array}{llr}\text { Hybrid enzyme } & \text { Subunits modified } & \begin{array}{r}\text { activity } \\ (\%)\end{array} \\ \text { HG1005 } \times \text { PL1138 } & \text { None } & 100 \\ & \text { PL1138 } & 0 \\ & \text { HG1005 } & 84 \\ \text { HG1005 } \times \text { PL1138 } & \text { None } & 0 \\ & \text { PL1138 } & 100 \\ & \text { HG1005 } & 0 \\ \text { PL1105 } \times \text { PL1138 } & \text { HG1005 and PL1138 } & 78 \\ & \text { PL1138 } & 0 \\ & \text { PL1105 } & 100 \\ \text { HG1019 } \times \text { PL1138 } & \text { PL1105 and PL1138 } & 0 \\ & \text { None } & 90 \\ & \text { PL1138 } & 0 \\ & \text { HG1019 } & 100 \\ \text { PL1072 } \times \text { PL1138 } & \text { HG1019 and PL1138 } & 0 \\ & \text { None } & 75 \\ & \text { PL1138 } & 0 \\ & \text { PL1072 } & 100 \\ & \text { PL1072 and PL1138 } & 0 \\ & & 81 \\ & & 0\end{array}$

\section{DISCUSSION}

Complementation in vitro was observed only by denaturing and renaturing mixed extracts of a CRM ${ }^{+}$guaB mutant and PL1138 (guaB105). The lack of complementation by $\mathrm{CRM}^{-}$strains may arise because these mutants produce altered IMP dehydrogenase proteins which are susceptible to proteolysis and are therefore absent from cell-free extracts (Greeb et al., 1971; Schlesinger, 1974), although in some strains (e.g. HG1040) the mutations may prevent transcription and/or translation of guaB. The requirement for denaturing conditions indicates that the mutant strains produce IMP dehydrogenase in an aggregated form. For hybridization, the proteins must first be dissociated so that free monomers from different molecules can come together.

Intrallelic complementation amongst guaB mutants of $S$. typhimurium has been demonstrated by Schafer et al. (1974). The complementation between guaB mutants of $E$. coli likewise appears to be interallelic which implies that the subunits of IMP dehydrogenase are identical. The activity of the hybrid enzyme depends on which guaB mutant is paired with PL1138. For intercistronic complementation the expected enzyme activities would be fairly constant, irrespective of mutant pairing, at about $50 \%$ of the control value. As the IMP dehydrogenase activity of the hybrids that we examined never approached $50 \%$ of the control, interallelic complementation is again signified. To explain these observations by intercistronic complementation would require the added assumption that the variable and low recovery of enzyme activity arises from different degrees of proteolysis of the enzyme monomers or from altered sensitivity of mutated enzymes to denaturing conditions. One mutation (guaB105) would correspond to one cistron whereas 16 mutations would be placed in the other. Two cistrons of approximately equal size [mol. wt of the two dissimilar subunits suggested by Powell (1973) are 60000 and 44000] would not be expected to differ so significantly in their susceptibility to mutation, but as guaB105 maps to the right of all complementing mutations (Fig. 1) an intercistronic boundary between guaB105 and guaB85 
is conceivable. However, interallelic complementation has been observed between mutations in the operator distal portion of $l a c Z$ and mutations mapping elsewhere in the gene (Ullman et al., 1967).

The hybrid enzyme produced by complementing PL1138 with HG1001 is more heat labile than that formed from PL1138 with HG1005, which, in turn, is more heat labile than native reconstituted enzyme. This strongly indicates interallelic complementation for IMP dehydrogenase rather than intercistronic complementation which would yield hybrid enzyme with the same heat stability as native enzyme. Interallelic complementation is also favoured by the findings that no $\mathrm{CRM}^{-}$mutation mapping operator-proximal to guaB85 complements guaB105, and guaB86 ( $\left.\mathrm{CRM}^{-}\right)$does not complement any mutation operator-proximal to guaB85. However, the situation could again be confused by intracellular proteolysis of mutant forms of the enzyme prior to hybridization. Taken together with the finding of Krishnaiah (1975) and Gilbert et al. (1979) that the subunits of purified IMP dehydrogenase are identical, the evidence presented in this report is most compatible with a system of interallelic complementation.

Why does only one mutation ( $\mathrm{guaB105}$ ) complement all other $\mathrm{CRM}^{+}$mutations? GuaB105 was induced by EMS whereas the others were induced by NG. However, as both mutagens act in a similar manner, they are unlikely to produce mutations of different complementing types. Attempts to isolate other mutants similar to PL1138 (guaB105) have so far proved unsuccessful. This may indicate that the replacement of only a small number of specific amino acids confers the phenotype characteristic of guaB105. Perhaps significantly, strain PL1138 shows slightly leaky growth on guanine-free media; leaky mutants most often provide the best complementation (Fincham et al., 1979).

Proteolytic enzymes cleave the monomer of IMP dehydrogenase into two polypeptides of unequal size (Gilbert et al., 1979). This indicates the presence of a region in the protein particularly susceptible to proteolysis, possibly a 'bridge' between two globular domains. Strain PL1138 may produce a protein with an amino acid substitution in the smaller domain and the complementary mutants may produce proteins with amino acid substitutions in the larger domain. Alternatively, complementation may be similar to that observed for certain glutamate dehydrogenase mutants of Neurospora crassa. This allosteric enzyme has two conformational states, active and inactive. A number of mutant enzymes, all frozen in the inactive state, when mixed with a mutant with a defect in the active site were able to change conformation and regain activity (Brett et al., 1976; Watson \& Wootton, 1978). Similarly, repairable variant enzymes having conformationally deformed active sites have also been observed for tryptophan synthetase of $E$. coli (Kida \& Crawford, 1974). By analogy with these examples, the unique guaB mutant PL1138 would produce enzyme frozen in an inactive conformation but capable of being repaired by other monomers defective at the active site. Covalent modification of the hybrid enzymes supports this conclusion by demonstrating that only the monomers from PL1138 are catalytically active in the hybrids. The 'mutual correction' theory of Crick \& Orgel (1964) offers a less satisfactory explanation for the type of complementation described here.

H. J. G. received a supporting grant from the Science Research Council.

\section{REFERENCES}

Brett, M., Chambers, G. K., Holder, A. A. \& FinchAm, J. R. S. (1976). Mutational amino acid replacements in Neurospora crassa NADPspecific glutamate dehydrogenase. Journal of Molecular Biology 106, 1-22.

Crick, F. H. C. \& Orgel, L. E. (1964). The theory of interallelic complementation. Journal of Molecular Biology 8, 161-165.
Fincham, J. R. S., DAY, P. R. \& RADFord, A. (1979). In Fungal Genetics, 4th edn, pp. 82-83. Oxford: Blackwell Scientific Publications.

Gilbert, H. J. \& Drabble, W. T. (1978). Activesite alkylation of IMP dehydrogenase from wildtype Escherichia coli $\mathrm{K} 12$ and some guaB mutants. Proceedings of the Society for General Microbiology $5,49-50$. 
Gilbert, H. J., Lowe, C. R. \& Drabble, W. T. (1978). The subunit structure of IMP dehydrogenase of Escherichia coli K12. Proceedings of the Society for General Microbiology 5, 23.

Gilbert, H. J., Lowe, C. R. \& Drabble, W. T. (1979). Inosine 5'-monophosphate dehydrogenase of Escherichia coli : purification by affinity chromatography, subunit structure and inhibition by guanosine 5'-monophosphate. Biochemical Journal 183, 481-494.

Greeb, J., Atkins, J. F. \& Loper, J. C. (1971). Histidinol dehydrogenase (hisD) mutants of Salmonella typhimurium. Journal of Bacteriology 106, 421-431.

Kida, S. \& Crawford, I. P. (1974). Complementation in vitro between mutationally altered $\beta_{2}$ subunits of Escherichia coli tryptophan synthetase. Journal of Bacteriology 118, 551-559.

KRISHNAIAH, K. V. (1975). Inosinic acid 5'-monophosphate dehydrogenase from Escherichia coli: purification by affinity chromatography and some properties. Archives of Biochemistry and Biophysics 170, 567-575.

Lambden, P. R. \& Drabble, W. T. (1973). The gua operon of Escherichia coli K-12: evidence for polarity from guaB to guaA. Journal of Bacterio$\log y$ 115, 992-1002.

MacPhee, D. G. \& Stocker, B. A. D. (1969). Suppression of amber and ochre mutants in Salmonella typhimurium by a mutant $\mathrm{F}^{\prime}$-1-gal factor carrying an ochre suppressor gene. Journal of Bacteriology 100, 240-246.

Mager, J. \& Magasanik, B. (1960). Guanosine 5 '-phosphate reductase and its role in the interconversion of purine nucleotides. Journal of Biological Chemistry 235, 1474-1478.
NiJkamp, H. J. J. \& DeHaAN, P. G. (1967). Genetic and biochemical studies of the guanosine $5^{\prime}$ monophosphate pathway in Escherichia coli. Biochimica et biophysica acta 145, 31-40.

Powell, G. F. (1973). Subunit structure of inosinic acid dehydrogenase from Escherichia coli. Biochemistry 12, 1592-1595.

Schafer, M. P., Hannon, W. H. \& Levin, A. P. (1974). In vivo and in vitro complementation between guaB and in vivo complementation between guaA auxotrophs of Salmonella typhimurium. Journal of Bacteriology 117, 1270-1279.

Schlesrnger, M. J. (1974). Variants of Escherichia coli alkaline phosphatase: an example of the role of genetics in a study of protein structure and function. Biochemical Society Transactions 2, $827-831$.

Ullman, A., JacoB, F. \& Monod, J. (1967). Characterisation by in vitro complementation of a peptide corresponding to an operator-proximal segment of the $\beta$-galactosidase structural gene of Escherichia coli. Journal of Molecular Biology 24, 339-343.

VoGel, H. J. \& Bonner, D. M. (1956). Acetylornithinase of Escherichia coli: partial purification and some properties. Journal of Biological Chemistry 218, 97-106.

Watson, D. H. \& Wootton, J. C. (1978). Subunit ratios of separated hybrid hexamers of Neurospora NADP-specific glutamate dehydrogenase containing complementary mutationally modified monomers. Biochemical Journal 175, 1125-1133.

ZABIN, I. \& Villarejo, M. R. (1975). Protein complementation. Annual Review of Biochemistry 44, 295-313. 